\title{
ON THE UNEVEN DISTRIBUTION OF THE COPELATA OF THE ALCATRAZES AREA
}

\author{
(With a map and one table)
}

\author{
T. K. S. Björnberg \& L. Forneris *
}

The present paper deals with the Copelata collected during the voyage of the yacht "Igaraty", owned by Dr. Adalberto Ferreira do Valle, who kindly put it at the service of the Instituto Oceanografico of the University of São Paulo, from June 26 to June 29, 1954. The samples were collected by Prof. W. Bernard and Dr. Ingvar Emílsson. On Table 1 is the list of stations, their position, dates, number and percentage of the species in each sample as well as the characteristic data of the water of the surveyed area. All the plankton samples were collected with a standard net (Sverdrup et al, 1954, p. 379, fig. 91-d).

The percentage composition of the more frequent species at each station is given in Fig. 1. At all the stations (excepting station 10) Haplopleura longicauda was the most abundant species. Oikopleura fusiformis and Fritillaria pellucida were the next in abundance.

The observation of the hydrographical data (as Dr. I. Emílsson personally informed us), showed that the surveyed area was under the influence of three water masses: A - coastal waters (of low salinity and medium temperature ); B - northern waters (of high salinity and high temperature); C - deep water (of medium salinity and low temperature).

The comparison of our own with the hydrographical data shows that stations six, seven and nine, characterized by a large frequency of Oikopleura rufescens, were strongly influenced by northern waters and that stations five and eight, characterized by a large percentage of Haplopleura longicauda, Oikopleura fusiformis and Fritillaria pellucida were situated in mixed waters. The small differences in the percentages of species in these last two stations may be due to the fact that the collections were made at different hours of the day. Stations one, ten and eleven were under the influence of coastal waters. At station ten, the great

* Partially supported by a grant in aid of the National Research Council of Brazil (C. N. Pq.). 
abundance of $F$. haplostoma may be explained by a swarm of this species, a fact commonly observed in superficial coastal waters (Tokioka - 1951, p. 18 \& 1955, p. 253). Stations ten and eleven also show such a small influence of northern waters (see Fig. 1) that 0 . rufescens there occurred in insignificant quantities.

The inversion in the frequency of $H$. longicauda and $O$. fusiformis in the samples from station ten may be due to the different hours in which the collecting was made.

It is strange that $O$. dioica was not found in pure coastal water. This neritic, euryhaline and eurythermic species seems to avoid certain types of coastal waters (Russell \& Colman, 1935, p. 228). We may conclude that the northern waters of this region were characterized in these samples by the presence of o. rufescens; the coastal waters, by the presence of $F$. pellucida; and that $F$. haplostoma was found there in swarms. We did not consider the influence of the thermoclyne to be significant in stations five and nine where it was observed at $40 \mathrm{~m}$ and at $20 \mathrm{~m}$ depth respectively because the quantity of water filtered by the net under the thermoclyne was very small when compared to the quantity of water filtered above it, so that it was here considered to be irrelevant.

It may finally be added that the occurrence of Tectillaria fertilis (station eight) in our samples widens the southern boundary of the distribution of this species, known up to now from $15^{\circ}$ North upwards in the Atlantic Ocean (Lohmann, 1896, p. 30).

\section{S U M A R I O}

Neste trabalho são estudados os Copelata coletados durante a viagem do "Igaraty" à Ilha de Alcatrazes. A lista das estações, sua localização, as datas e outros dados sôbre as águas da área pesquisada, assim como o número e a porcentagem de ocorrência de cada espécie em cada amostra estão na Tabela 1. Tôdas as coletas foram feitas com uma rêde "standard" (Sverdrup et al-1954, p. 379, fig. 91-d). Comparando os dados hidrográficos, fornecidos por informação pessoal do $\mathrm{Dr}$. I. Emílsson, com os nossos, podemos concluir que, nestas amostras, as águas vindas do norte (vide estações 6,7 e 9) são caracterizadas pela presença de $O$. rufescen. em grande abundância; as águas costeiras, pela presença de $F$. pellucida (vide estações 1, 10 e 11) e as águas misturadas (vide estações 5 e 8, Tabela 1 e Figura 1) são caracterizadas pela ocorrência de H. longicauda, O. fusiformis e F. pellucida em freqüências elevadas. Houve ocorrência ainda de "nuvens" de F. haplostoma na estação 10, fato comum em águas costeiras e superficiais do Japão (Tokioka, 1951 e 1955).

A influência da termoclina observada nas estações 5 e 9 não foi considerada significativa pois foi pequena a quantidade de água atravessada pela rêde abaixo dela, em comparação com a grande quantidade ocorrida acima da mesma. $O$ aparecimento de Tectillaria fertilis aumenta o limite sul de distribuição desta espécie no Atlântico, pois era conhecida apenas nas correntes quentes do hemisfério norte dêste oceano (Lohmann, 1896, p. 30).

\section{ACKNOWLEDGEMENTS}

Thanks are due to Dr. I. Emílsson for furnishing us with the valuable hydrographical data. To him, to Dr. M. Vannucei and Mr. João de Paiva Carvalho, for the eriticism of the manuscript and helpful suggestions. To the Rockefeller Foundation we are indebted for granting us some of the equipment used. 


\section{REF E R E N C S}

LOHMANN, H., - 1896. "Die Appendicularien der Plankton Expedition". Ergeb. Plankton Exped., Vol. 2 F. c. - p. 1-148, 20 taf., 3 cart., 1 diagr.

RUSSELL, F. S. \& COLMAN, J. S., - 1935. The Zooplankton IV. The occurrence \& seasonal distribution of the Tunicata, Mollusea \& Coelenterata. Great Barrier Reef Exp. 1928-29. Vol. 2 (7), p. 219-231.

SVERDRUP, H., JOHNSON, M. W. \& FLEMING, R. H., - 1954. The Oceans. $\mathrm{X}+1087$ p. Prentice-Hall, Inc. New York.

TOKIOKA, T., - 1951. Pelagic Tunicates \& Chaetognaths collected during the cruises to the new Yamato Bank in the Sea of Japan. Publ. Seto Mar. Biol. Lab., Vol. II, n. ${ }^{\circ}$ 1, p. 1-25.

- 1955. General considerations on Japanese Appendicularia. Publ. Seto Mar. Biol. Lab., Vol. IV (2-3), p. 251-261. 


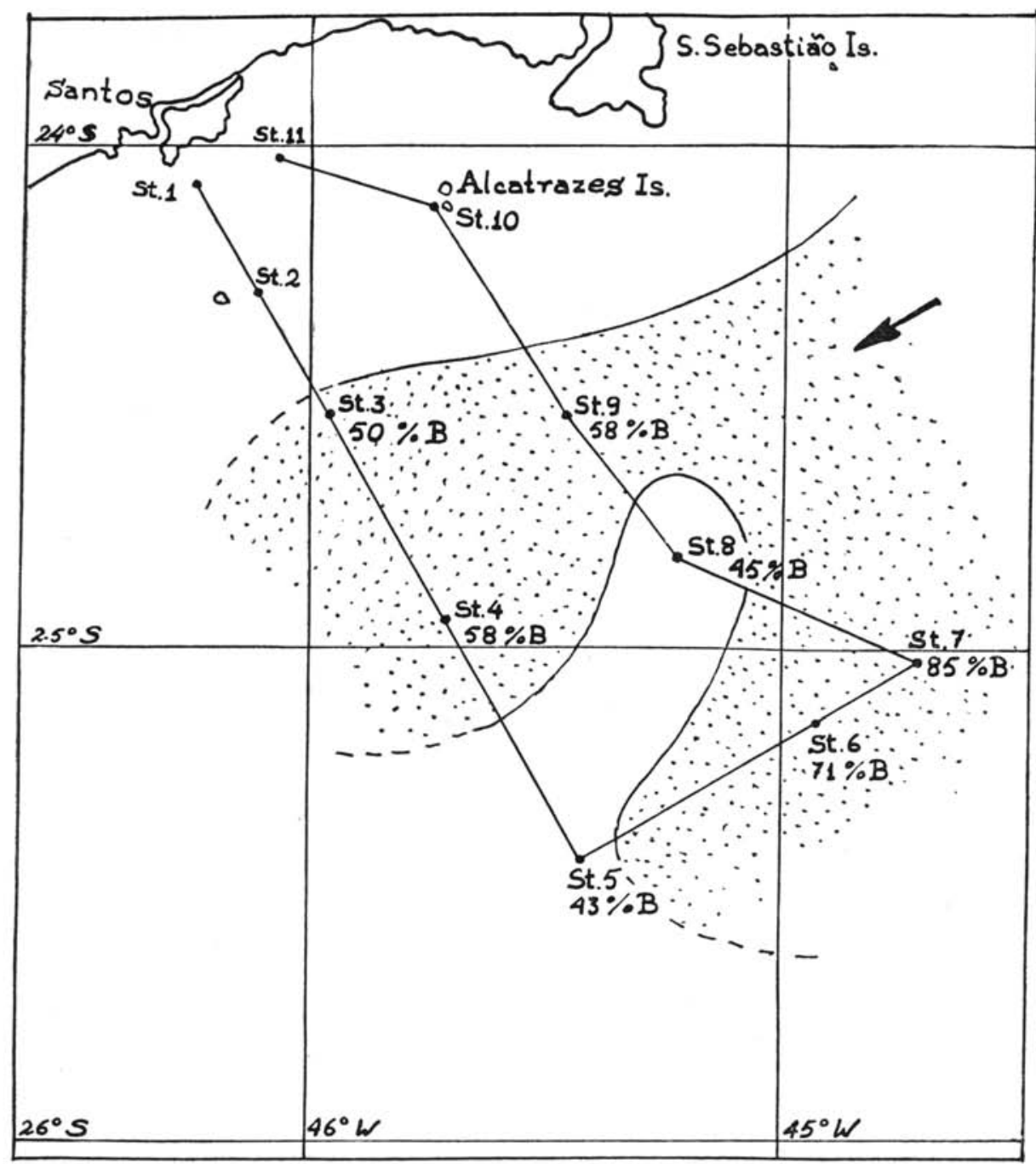

Northern Waters (B)

Map of the Alcatrazes Area, with position of stations and approximate distribution of northern water mass from April 26 to April 29, 1954 ("Igaraty" trip). 
F I o u $P$ I.

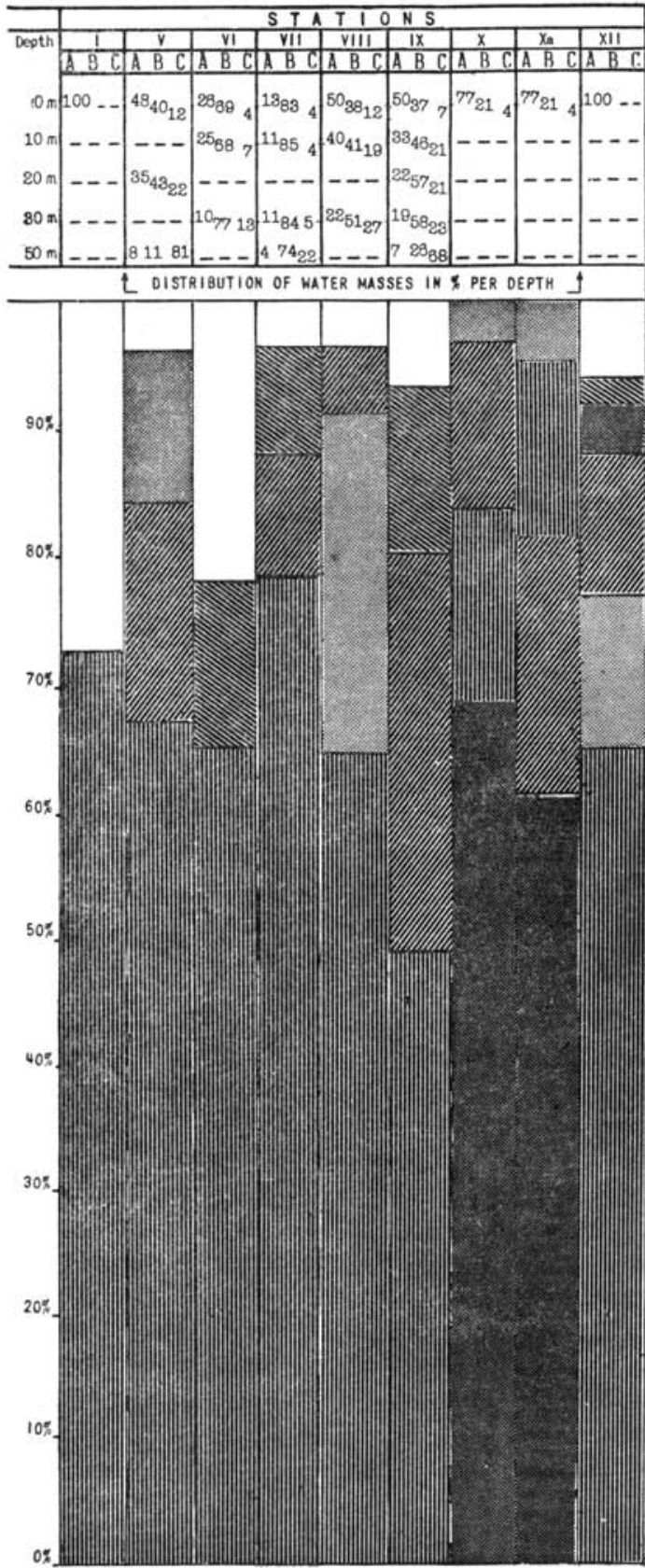

PERCENTAGES OF SPECIES PER STATIOK
CHARACTERISTICS

OF WATER MASSES:

A: $\approx 1 . \kappa_{0}=33.5$

cemp. 21.6

(COASTAL Wateg)

sel. 24.0

(Nortacser wates)

C: sal. $\%_{0}=35.6$

temp. $=15.4$

(oEer vater)

SPECIES:

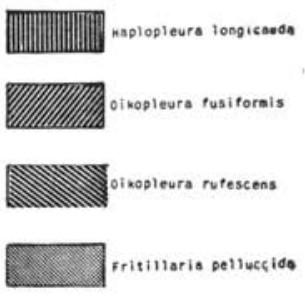

fritillaria haplostoas

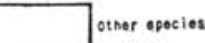




\begin{tabular}{|c|c|c|c|c|c|c|c|c|c|}
\hline 商 & $\Rightarrow$ & E & $\approx$ & $\approx$ & 羿 & 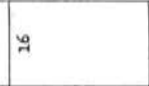 & $\frac{7}{2}$ & $\frac{6}{-7}$ & 营 \\
\hline 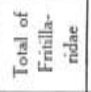 & $\circ$ & $a$ & m & n & $m$ & -4 & 照 & \% & 䇣 \\
\hline 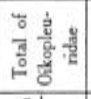 & A & 8 & 8 & 8 & ‡ & $\approx$ & $\begin{array}{l}\text { aे } \\
\text { in } \\
\text { a }\end{array}$ & $\cong$ & $\frac{\$}{3}$ \\
\hline 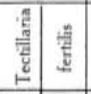 & ' & , & . & . & $-\frac{\bar{G}}{\dot{g}}$ & . & . & ' & ' \\
\hline म & ' & , & . & . & . & 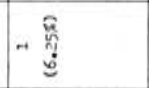 & . & ' & , \\
\hline 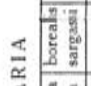 & ' & 1 & . & 1 & . & , & 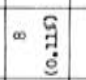 & $\sim$ ă & 1 \\
\hline 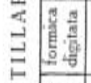 & ' & - & $-\frac{9}{3}$ & $\begin{array}{r}-6 \\
-19 \\
\end{array}$ & . & i &. & . & 1 \\
\hline 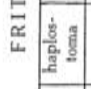 & ' & ' & - 魷 & . & . & , & 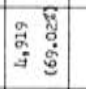 & 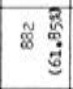 & ज魷 \\
\hline \begin{tabular}{|l|} 
题 \\
営 \\
\end{tabular} & ' & 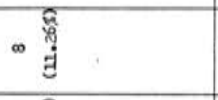 & $-\frac{5}{3}$ & 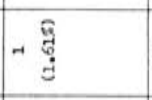 & Ұ & ' & 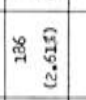 & «员 & 亭 \\
\hline 总 & $m \frac{\sqrt[6]{4}}{5}$ & N & ' & ' & ' & N & . & ' & 量 \\
\hline 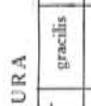 & ' & ' & +亳 & ' & , & , & . & . & , \\
\hline $\begin{array}{ll} \\
\end{array}$ & 1 & ' & $+\frac{5}{3}$ & ' & $\approx$ & ' & . & ' & ' \\
\hline \begin{tabular}{|l|l|}
\multicolumn{2}{|c|}{} \\
\\
\end{tabular} & ' & ' & 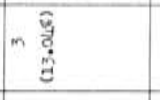 & 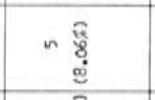 & 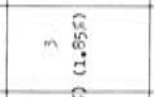 & \% & 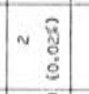 & N & 을 \\
\hline 辛曾 & ' & 요용 & . & $\begin{array}{r}\frac{\pi}{8} \\
\qquad \frac{8}{8} \\
\end{array}$ & $\infty \frac{3}{8}$ & 的苟 & 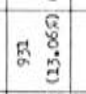 & 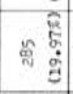 & 总 \\
\hline 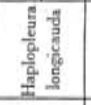 & $\infty \frac{\sqrt{2}}{E}$ & $\begin{array}{l}5 \\
9 \\
5 \\
5\end{array}$ & $\approx$ त & 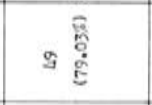 & 声爱 & $\infty \frac{\sqrt{8}}{8}$ & 然 & 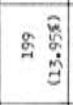 & 㜊 \\
\hline 首上 & 8 & กี & $\frac{12}{8}$ & 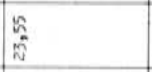 & 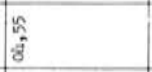 & $\frac{n}{2}$ & $\begin{array}{l}2 \\
2 \\
7 \\
7\end{array}$ & $\begin{array}{l}R \\
0 \\
0\end{array}$ & $\dddot{7}$ \\
\hline 点。 & 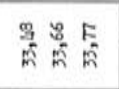 & 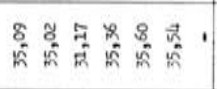 & 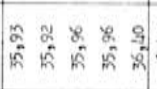 & 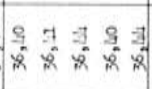 & 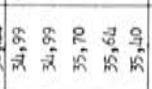 & 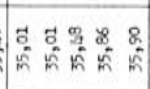 & 월 & & 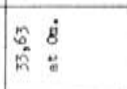 \\
\hline U & กี & 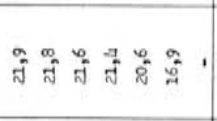 & 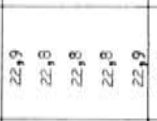 & 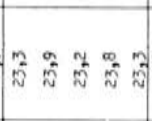 & 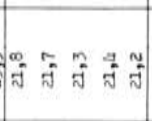 & 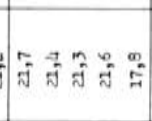 & $\stackrel{4}{\infty}$ & & 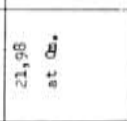 \\
\hline 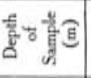 & $\circ \approx$ & 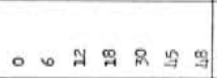 & $0 \begin{array}{llll}0 & 0 & 9 & \cdots\end{array}$ & 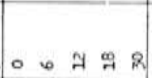 & $\cong$ & $\circ \odot \approx \cong \Omega$ & m. & & $\begin{array}{l}4 \\
3 \\
3 \\
8\end{array}$ \\
\hline $7 \cap \vee \mathrm{B}$ & TVOISHEA & 7VכLLUสA & TVOILUSA & 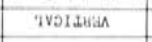 & IVOTLËA & IVJILdaA & TVLNOZZ & 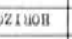 & 7V.2LO25 \\
\hline 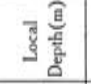 & $\exists$ & 8 & ఫ & $\underset{-2}{*}$ & $\approx$ & $\$$ & ' & & 1 \\
\hline 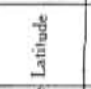 & 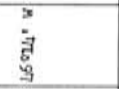 & $\begin{array}{l}x \\
0 \\
0 \\
0 \\
0\end{array}$ & 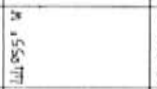 & 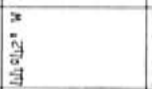 & 落 & $\begin{array}{l}x \\
x \\
5 \\
0 \\
9 \\
g\end{array}$ & 药 & & $\begin{array}{l}3 \\
3 \\
8 \\
3 \\
3\end{array}$ \\
\hline 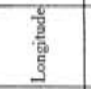 & ¿ & "ू. & 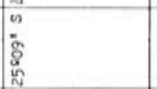 & 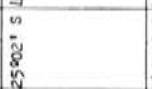 & 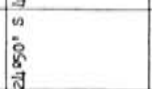 & $\frac{\sqrt{3}}{2}$ & 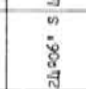 & & a \\
\hline ะั & 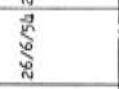 & 荵 & 离 & 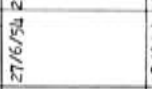 & 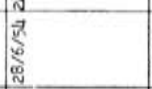 & 竞 & 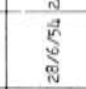 & & 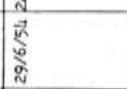 \\
\hline 量 & - & n & 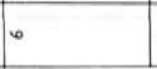 & r & $\infty$ & $\alpha$ & 웅 & & F \\
\hline
\end{tabular}

